\title{
$B$ DECAYS TO OPEN AND HIDDEN CHARM AT BABAR
}

\author{
STEFANIA RICCIARDI \\ Royal Holloway, University of London, Egham Hill, Egham, Surrey, TW20 0EX, UK \\ E-mail:s.ricciardi@rhul.ac.uk
}

\begin{abstract}
A wealth of new measurements of hadronic $B$ decays to open charm and charmonium have been performed on the data sample collected by the BaBar experiment at the PEP-II $e^{+} e^{-} B$-factory. We report the first measurement of the rates for inclusive charm production separately for charged and neutral $B$ mesons. Combining measurements of the decay rate of $B^{0} \rightarrow D_{s}^{*+} D^{*-}$ obtained with a partial reconstruction technique and the complete reconstruction of $B^{0} \rightarrow D_{s}^{*+} D^{*-}$ with $D_{s}^{+} \rightarrow \phi \pi^{+}$, we obtain the most precise measurement of $\mathcal{B}\left(D_{s}^{+} \rightarrow \phi \pi^{+}\right)$. We present an update of the measurements of the branching fractions of exclusive $B$ decays to $J / \psi, \psi(2 S), \chi_{c 1}, \chi_{c 2}$ and a kaon or a $K^{*}$. In addition, we show preliminary results on exclusive decay rates to final states with baryons, or newly-discovered particles, such as $D_{s J}^{*}(2317), D_{s J}(2460)^{+}$, and the $X(3872)$.
\end{abstract}

\section{Introduction}

The successful performance of the $B$ factories enables the study of $B$ meson decays with unprecedented sensitivity. In the following, we report the new results on the decay rates of $B$ mesons to final states including a charmed meson, a charmed baryon, or a charmonium resonance. These studies are performed with various reconstruction techniques to exploit the potential of about $10^{8}$ million $B \bar{B}$ pairs collected with the BaBar detector at the PEP-II $e^{+} e^{-}$collider in the years 1999-2003.

\section{Branching fraction of inclusive $B^{-}$and $\bar{B}^{0}$ decays to \\ flavour-tagged $D, D_{s}^{+}$, and $\Lambda_{c}$}

We have measured for the first time the branching fractions for inclusive decays of $B$ mesons to $D^{0}, \bar{D}^{0}, D^{+}, D^{-}, D_{s}^{+}, D_{s}^{-}$, $\Lambda_{c}^{+}$, and $\bar{\Lambda}_{c}^{-}$, separately for neutral and charged $B$ mesons. The measurements, based on a sample of 88.9 million $B \bar{B}$ events are reported in ref. ${ }^{1}$.

Events are selected by completely reconstructing one $B$ and searching for a reconstructed charmed particle in the rest of the event. The numbers of charmed particles per $B^{-}$decay $\left(n_{c}{ }^{-}=1.313 \pm 0.037 \pm 0.062_{-0.042}^{+0.063}\right)$ and per $\bar{B}^{0}$ decay $\left(n_{c}{ }^{0}=1.276 \pm 0.062 \pm\right.$ $\left.0.058_{-0.046}^{+0.066}\right)$ are consistent with previous measurements and with theoretical expectations ${ }^{a}$. The errors reflect the statistical, the systematic, and the branching-fraction uncertainties.

\section{Partial Reconstruction of $B^{0} \rightarrow D_{s}^{*+} D^{*-}$ and measurement of $D_{s}^{+} \rightarrow \phi \pi^{+}$}

The aim of this analysis ${ }^{3}$ is to measure the branching fractions $\mathcal{B}\left(B^{0} \rightarrow D_{s}^{*+} D^{*-}\right)$ and $\mathcal{B}\left(D_{s}^{+} \rightarrow \phi \pi^{+}\right)$. A precise measurement of the branching fraction for the $D_{s}^{+} \rightarrow$ $\phi \pi^{+}$mode is important because nearly all $D_{s}^{+}$branching fractions are determined by normalizing the measurements to $\mathcal{B}\left(D_{s}^{+} \rightarrow\right.$ $\left.\phi \pi^{+}\right)$, and, therefore, its present uncertainty of about $25 \%^{2}$ affects many of the results regarding $D_{s}^{+}$mesons.

The $B^{0} \rightarrow D_{s}^{*+} D^{*-} \rightarrow\left(D_{s}^{+} \gamma\right)\left(\bar{D}^{0} \pi^{-}\right)$ decay is reconstructed using two different methods. The first method uses a partial reconstruction technique, in which only the $D^{*-}$ is fully reconstructed, and then combined with the soft photon from the $D_{s}^{*+} \rightarrow D_{s}^{+} \gamma$ decay, without requiring explicit $D_{s}^{+}$reconstruction. The missing mass distribution is shown in Figure 1. Using this technique, we measure $\mathcal{B}\left(B^{0} \rightarrow D_{s}^{*+} D^{*-}\right)=$

${ }^{a}$ The inclusion of charge conjugation modes is implied throughout this report. 


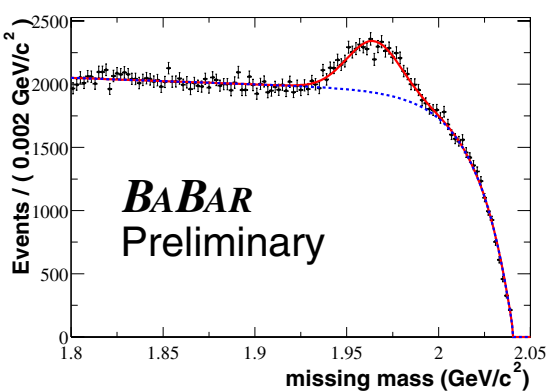

Figure 1. Missing mass distribution in the inclusive reconstruction of $B^{0} \rightarrow D_{s}^{*+} D^{*-}$.

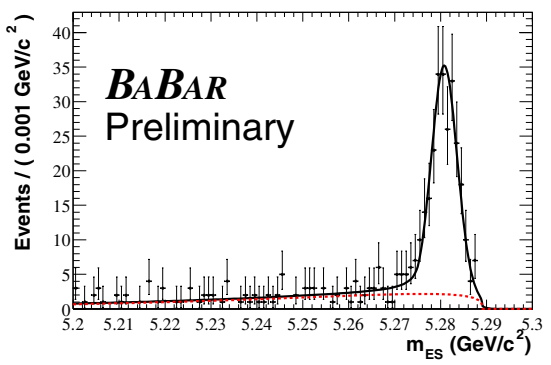

Figure 2. Exclusive reconstruction of $B^{0} \rightarrow$ $D_{s}^{*+} D^{*-}$. Fit to the total data sample overlaid to data.

$(1.85 \pm 0.09 \pm 0.16) \%$.

In the second method the whole decay chain of $B^{0} \rightarrow D_{s}^{*+} D^{*-}$ is reconstructed and the product of branching fractions $\mathcal{B}\left(B^{0} \rightarrow\right.$ $\left.D_{s}^{*+} D^{*-}\right) \times \mathcal{B}\left(D_{s}^{+} \rightarrow \phi \pi^{+}\right)$is measured. We use only $D_{s}^{+} \rightarrow \phi \pi^{+}$decays where the $\phi$ meson is reconstructed in the $K^{+} K^{-}$channel. The number of events is extracted from a fit to the beam-energy substituted mass of the $B, m_{\mathrm{ES}}{ }^{b}$, and is shown in Fig. 2. Comparing these two measurements provides a modelindependent determination of the $\mathcal{B}\left(D_{s}^{+} \rightarrow\right.$ $\phi \pi^{+}$) branching fraction. We obtain the following preliminary results:

$$
\mathcal{B}\left(D_{s}^{+} \rightarrow \phi \pi^{+}\right)=(4.71 \pm 0.47 \pm 0.35) \% .
$$

${ }^{b} m_{\mathrm{ES}}=\sqrt{E_{\text {beam }}^{* 2}-p_{B}^{* 2}}$, where $E_{\text {beam }}^{*}$ is the beam energy and $p_{B}^{*}$ is the reconstructed $B$ momentum in the center of mass frame.

\section{Branching fractions of}

$$
\begin{aligned}
& B^{0} \rightarrow D^{*-} p \bar{p} \pi^{+}, B^{0} \rightarrow D^{-} p \bar{p} \pi^{+}, \\
& B^{0} \rightarrow \bar{D}^{* 0} p \bar{p}, \text { and } B^{0} \rightarrow \bar{D}^{0} p \bar{p} .
\end{aligned}
$$

$B$ meson decays to final states which include a charmed meson and a baryon anti-baryon pairs are fully reconstructed in a sample of 124 million $B \bar{B}$ pairs. The decay of $B^{0} \rightarrow$ $D^{-} p \bar{p} \pi^{+}$has been observed for the first time with a measured branching fraction $\mathcal{B}\left(B^{0} \rightarrow\right.$ $\left.D^{-} p \bar{p} \pi^{+}\right)=(3.80 \pm 0.35 \pm 0.46) \times 10^{-4}$. The following branching fractions are also determined: $\mathcal{B}\left(B^{0} \rightarrow D^{*-} p \bar{p} \pi^{+}\right)=(5.61 \pm 0.59 \pm$ $0.73) \times 10^{-4}, \mathcal{B}\left(B^{0} \rightarrow \bar{D}^{* 0} p \bar{p}\right)=(0.67 \pm$ $0.21 \pm 0.09) \times 10^{-4}$, and $\mathcal{B}\left(B^{0} \rightarrow \bar{D}^{0} p \bar{p}\right)=$ $(1.24 \pm 0.14 \pm 0.12) \times 10^{-4}$. Plots can be found in ref. ${ }^{6}$.

\section{Exclusive decays to charmonium and a kaon or $K^{*}$}

The dynamics of these color-suppressed decays is expected to be highly influenced by strong interactions effects. The measurements of the branching fractions of factorization-allowed two-body decays to charmonium $\left(J / \psi / \psi(2 S) / \chi_{c 1}\right)$ and a kaon or $K^{*}$ have been updated with a sample of 124 million $B \bar{B}$ pairs. The results can be found in ref. ${ }^{4}$. Our results for $J / \psi$ and $\psi(2 S)$ are in good agreement with previous measurements and have comparable or superior precision. Our $\chi_{c 1}$ results have much better precision and the $B^{+} \rightarrow \chi_{c 1} K^{*+}$ branching fraction was measured for the first time. Assuming isospin invariance, we find the ratio of charged to neutral $B$ meson production on the $\Upsilon(4 \mathrm{~S})$ resonance to be compatible with unity within two standard deviations. No direct $C P$ violation has been observed from the measurements of charge asymmetries, as we found them to be compatible with zero.

The factorization-suppressed decays to $\chi_{c 2}$ and a kaon or $K^{*}$ are also searched for in the same data sample. No significant sig- 
nal is found and improved upper limits on the decay are set ${ }^{5}$.

\section{Spectroscopy studies}

\subsection{Studies of the $B \rightarrow D_{s J} D^{(*)}$ decays}

The recent discovery of two narrow resonances, $D_{s J}^{*}(2317)$ and $D_{s J}(2460)^{+}$, at mass values lower than the expected for $J^{P}=0^{+}$and $1^{+}$charmed-strange mesons, poses several questions regarding their properties. To establish their nature, the production of $D_{s J}^{*}(2317)$ and $D_{s J}(2460)^{+}$in $B$ decay in conjunction with a $D$ meson or a $D^{*}$ is studied. Studies are based on 122 million $B \bar{B}$ events, where one of the $B$ meson is fully reconstructed in the $D_{s J}^{*}(2317) D^{(*)-}\left(D_{s J}^{*}(2317)^{+} \rightarrow D_{s}^{+} \pi^{0}\right)$ or in the $D_{s J}(2460)^{+} D^{(*)-}\left(D_{s J}(2460)^{+} \rightarrow\right.$ $\left.D_{s}^{+} \gamma, D_{s}^{+} \pi^{0}\right)$ decay channel. The determined branching fractions can be found in ref. ${ }^{7}$.

In addition, an angular analysis of the decay $B \rightarrow D_{s J}(2460)^{+} \bar{D}$ with $D_{s J}(2460)^{+} \rightarrow D_{s}^{+} \gamma$ is performed. The result excludes $J^{P}=2^{+}$and supports the hypothesis that the $D_{s J}(2460)^{+}$is a $J^{P}=1^{+}$state.

\subsection{The X(3872)}

The observation of $X(3872) \rightarrow J / \psi \pi^{+} \pi^{-}$by Belle, CDF, and D0 8 has been confirmed by BABAR ${ }^{9}$. The signal peak in the $m_{\mathrm{ES}}$ distribution shown in Fig. $3(\mathrm{~b})$ is due to selected candidates in a window of $\pm 10 \mathrm{MeV} / \mathrm{c}^{2}$ around the expected mass of the $X(3872)$. We measure the branching fraction product, $\mathcal{B}\left(B^{-} \rightarrow X(3872) K^{-}\right) \times \mathcal{B}\left(B^{-} \rightarrow\right.$ $\left.X(3872) K^{-}\right)=(1.28 \pm 0.41) \times 10^{-5}$. Since the measured $X(3872)$ mass is very close to the $D^{* 0} \bar{D}^{0}$ mass threshold, a molecular model ${ }^{10}$ has been suggested among the numerous theoretical explanations on the nature of this new particle. If a charm molecule exists, then bound states of charged and neutral charmed-meson or charged molecular states

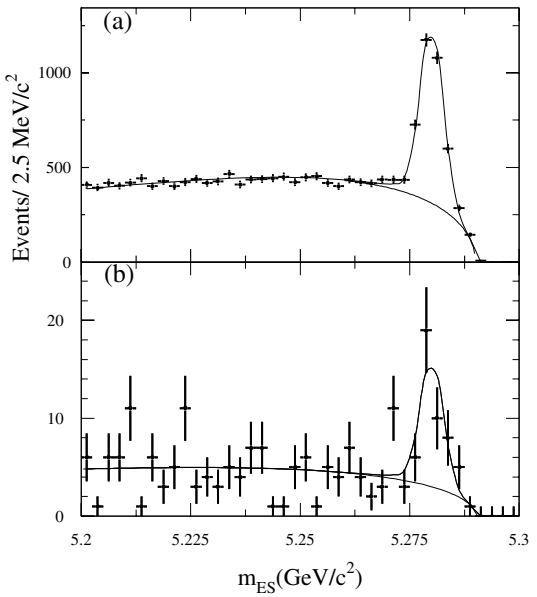

Figure 3. Distribution of $m_{\mathrm{ES}}$ for a) $B \rightarrow J / \psi \mathrm{K} \pi^{+} \pi^{-}$candidates, and b) events with $3862<m\left(J / \psi \pi^{+} \pi^{-}\right)<3882 \mathrm{MeV} / c^{2}$.
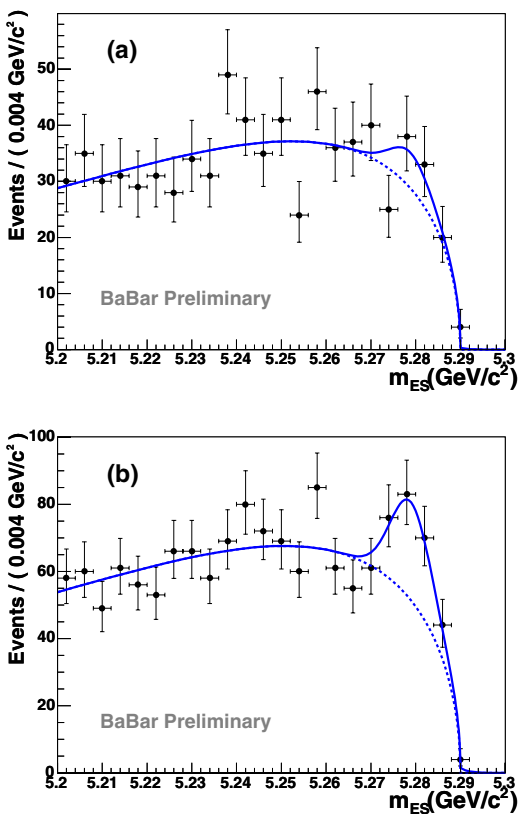

Figure 4. Fitted $m_{\mathrm{ES}}$ distribution for $B^{0} \rightarrow X^{ \pm} K^{\mp}$ with the $X^{ \pm}$signal region selection, $\mid m\left(J / \psi \pi^{ \pm} \pi\right)$ $3872 \mathrm{MeV} / c^{2} \mid<12 \mathrm{MeV} / c^{2}$, (a), and with the sideband region selection, $48<\mid m\left(J / \psi \pi^{ \pm} \pi\right)$ $3872 \mathrm{MeV} / c^{2} \mid<72 \mathrm{MeV} / c^{2}$, (b). 
Table 1. The estimated number of signal events, $90 \%$ C.L. upper limit of signal events, the branching fraction upper limits, and the branching fraction $(\mathcal{B})$ for $B$ decay to $X(3872){ }^{ \pm} K$.

\begin{tabular}{|l|c|c|c|c|}
\hline Mode & $n_{s} \pm \sigma_{s}$ & $N_{90}$ & $90 \%$ C.L. & $\mathcal{B}$ \\
\hline$J / \psi \pi^{ \pm} \pi^{0} K^{\mp}$ & $-14.8 \pm 13.9$ & 15.6 & $<5.8 \times 10^{-6}$ & $(-5.5 \pm 5.2) \times 10^{-6}$ \\
$J / \psi \pi^{ \pm} \pi^{0} K_{S}^{0}$ & $3.4 \pm 8.3$ & 15.9 & $<11 \times 10^{-6}$ & $(2.3 \pm 5.7) \times 10^{-6}$ \\
\hline
\end{tabular}

are plausible. In particular, if the observed decay is $J / \psi \rho^{0}\left(\rightarrow \pi^{+} \pi^{-}\right)$, then a charged partner, $X(3872)^{ \pm} \rightarrow J / \psi \rho^{ \pm}$may exist with favourable decay rate. We have searched for the charged partner in $B^{ \pm} \rightarrow X(3872){ }^{ \pm} K_{S}^{0}$ where $X^{ \pm} \rightarrow J / \psi \pi^{ \pm} \pi^{0}$, using 213 million $B \bar{B}$ events ${ }^{11}$. Events are fully reconstructed using standard criteria on track quality and kinematic cuts. The combinatorial background is estimated with a fit to the $m_{\mathrm{ES}}$ distribution of selected $B^{0}$ and $B^{+}$events in a region of $\pm 12 \mathrm{MeV} / c^{2}$ around the $X(3872)$ nominal mass, and is shown in Fig. 4(a) for $B^{0}$ candidates. A sideband region $48<\left|m\left(J / \psi \pi^{ \pm} \pi\right)-3872 \mathrm{MeV} / c^{2}\right|<$ $72 \mathrm{MeV} / c^{2}$ is used to estimate the background component which peaks at the $B$ mass in the $m_{\mathrm{ES}}$ distribution, and is shown in Fig. 4(b). The results are summarized in Table 1 . We see no significant excess neither for neutral, nor for charged $B$ mesons. Hence, our results do not support the existence of charged molecular states or charged partners of the $X(3872)$.

\section{Summary}

We have presented several new measurements of branching fractions of $B$ meson decays to open and hidden charm, which improve the precision of previous measurements or provide new insights on the spectroscopy of charm and charmonium systems. In addition, from the study of $B^{0} \rightarrow D_{s}^{*+} D^{*-}$, we have obtained the most precise determination of the $D_{s}^{+}$branching fraction to $\phi \pi^{+}, \mathcal{B}\left(D_{s}^{+} \rightarrow\right.$ $\left.\phi \pi^{+}\right)=(4.71 \pm 0.47$ (stat. $) \pm 0.35$ (syst. $\left.)\right) \%$. This measurement will have an impact on many other results involving the $D_{s}^{+}$meson.

\section{References}

1. The BABAR Collaboration, B. Aubert et al., hep-ex/0408113, sub. to Phys. Rev. D, and references therein.

2. The CLEO Collaboration, M. Artuso et al., Phys. Lett. B 378, 364 (1996).

3. The BABAR Collaboration, B. Aubert et al., hep-ex/0408040, contributed to ICHEP'04.

4. The BABAR Collaboration, B. Aubert et al., hep-ex $/ 0408036$, contributed to ICHEP'04.

5. The BABAR Collaboration, B. Aubert et al., hep-ex/0408033, contributed to ICHEP'04.

6. The BABAR Collaboration, B. Aubert et al., hep-ex/0408035, contributed to ICHEP'04.

7. The BABAR Collaboration, B. Aubert et al., hep-ex/0408041, to appear in Phys. Rev. Lett.

8. Belle Collaboration, S.K. Choi et al., Phys. Rev. Lett. 91, 262001 (2003); CDF Collaboration, Phys. Rev. Lett. 93 (2004) 072001; D0 Collaboration, accepted by Phys. Rev. Lett.

9. The BABAR Collaboration, B. Aubert et al., hep-ex/0406022.

10. N. Tornqvist, Phys. Lett. B 590, 209 (2004); M. B. Voloshin, Phys. Lett. B 579, 316 (2004); F. Close and P. Page, Phys. Lett. B 578, 119 (2004); C.Y. Wong, Phys. Rev. C 69, 055202 (2004); E. Braaten and M. Kusonai, Phys. Rev. D 69, 074004 (2004); E. Swanson, Phys. Lett. B 588, 189 (2004).

11. The BABAR Collaboration, B. Aubert et al., hep-ex/0408083. 